\title{
Corrigendum to "Biological Characteristics and Carrier Functions of Pegylated Manganese Zinc Ferrite Nanoparticles"
}

\author{
Ting Guo ${ }^{1},{ }^{1}$ Xiaofeng Dou, ${ }^{2}$ Mei Lin $\mathbb{D}^{3},{ }^{3}$ Junxing Huang, ${ }^{4}$ Chenglin Zhou, ${ }^{3}$ Jun Zhang, ${ }^{5}$ \\ Hong Yu $\mathbb{D}^{6},{ }^{6}$ Xingmao Jiang $\mathbb{D},{ }^{7}$ Jun Ye $\mathbb{}{ }^{1},{ }^{1}$ Yujuan Shi, ${ }^{1}$ Yanhong Xiao $\mathbb{D},{ }^{1}$ Xuefeng Bian, ${ }^{1}$ \\ Xiaoqian Feng, ${ }^{1}$ and $\mathrm{Ning} \mathrm{Xu}^{8}$ \\ ${ }^{1}$ Institute of Clinical Medicine, Taizhou People's Hospital Affiliated to Nantong University, Taizhou, Jiangsu 225300, China \\ ${ }^{2}$ Medical Image Center, Taizhou People's Hospital Affiliated to Nantong University, Taizhou, Jiangsu 225300, China \\ ${ }^{3}$ Clinical Laboratory, Taizhou People's Hospital Affiliated to Nantong University, Taizhou, Jiangsu 225300, China \\ ${ }^{4}$ Oncology Department, Taizhou People's Hospital Affiliated to Nantong University, Taizhou, Jiangsu 225300, China \\ ${ }^{5}$ Department of Nuclear Medicine, Taizhou People's Hospital Affiliated to Nantong University, Taizhou, Jiangsu 225300, China \\ ${ }^{6}$ Pathology Department, Taizhou People's Hospital Affiliated to Nantong University, Taizhou, Jiangsu 225300, China \\ ${ }^{7}$ Hubei Key Lab of Novel Reactor \& Green Chemical Technology, Key Laboratory for Green Chemical Process of Ministry \\ of Education, School of Chemical Engineering and Pharmacy, Wuhan Institute of Technology, Wuhan 430205, China \\ ${ }^{8}$ Gastrointestinal Surgery, Taizhou People's Hospital Affiliated to Nantong University, Taizhou, Jiangsu 225300, China
}

Correspondence should be addressed to Mei Lin; 1_mei@163.com

Received 14 January 2020; Accepted 16 January 2020; Published 8 April 2020

Copyright $\odot 2020$ Ting Guo et al. This is an open access article distributed under the Creative Commons Attribution License, which permits unrestricted use, distribution, and reproduction in any medium, provided the original work is properly cited.

In the article titled "Biological Characteristics and Carrier Functions of Pegylated Manganese Zinc Ferrite Nanoparticles" [1], there was a spelling error in author Xiaofeng Dou's name in the author list. This is corrected as shown above.

\section{References}

[1] T. Guo, F. Dou, M. Lin et al., "Biological characteristics and carrier functions of pegylated manganese zinc ferrite nanoparticles," Journal of Nanomaterials, vol. 2019, Article ID 6854710, 10 pages, 2019. 